\section{Regards sur l'économie allemande}

Bulletin économique du CIRAC

115 | 2014

Varia

\title{
Réformer la gouvernance de l'UE après la crise
}

\section{Christian Lequesne}

\section{(2) OpenEdition \\ Journals}

Édition électronique

URL : http://journals.openedition.org/rea/4764

DOI : 10.4000/rea.4764

ISSN : 1965-0787

Éditeur

CIRAC

Édition imprimée

Date de publication : 1 décembre 2014

Pagination : 31-34

ISSN : 1156-8992

Référence électronique

Christian Lequesne, "Réformer la gouvernance de l'UE après la crise », Regards sur l'économie

allemande [En ligne], 115 | décembre 2014, mis en ligne le 01 décembre 2016, consulté le 04 mai 2019. URL : http://journals.openedition.org/rea/4764 ; DOI : 10.4000/rea.4764 


\section{Réformer la gouvernance de 1'UE après la crise}

\section{Christian Lequesne}

Les difficultés qu'a connues l'Union économique et monétaire (UEM) depuis 2008 ont révélé non seulement la fragilité économique de l'Union européenne (UE), mais aussi les faiblesses de son modèle de gouvernance. La sortie de crise pose plus que jamais la question du lien entre I'UEM et l'Union politique de l'Europe.

Cette question n'est pas neuve. Elle avait déjà été posée lors de la négociation du Traité de Maastricht (1992). Il faut se rappeler qu'à l'époque, le gouvernement d'Helmut Kohl avait insisté pour que la Conférence intergouvernementale chargée de négocier l'UEM - que François Mitterrand avait tant voulu - se double d'une Conférence intergouvernementale sur l'Union Politique (voir Doutriaux/Lequesne, 2013). Ce lien est revenu ensuite de manière récurrente du côté allemand, consacrant ce que les économistes allemands ont appelé dans les années 1990 la "théorie du couronnement de l'UEM » (Krönungstheorie ; Guérot, 2012). L'argument est que la monnaie unique ne peut exister vraiment que si elle est au service d'une union politique de l'Europe, marquée par un gouvernement fédéral européen et une nouvelle légitimité parlementaire. II s'agit en quelque sorte de la projection sur l'Union européenne de l'expérience fédérale de l'Allemagne.

Dans le contexte politique de 2015, il n'est guère difficile d'observer que la plupart des chefs d'Etat et de gouvernement ne souhaitent pas une nouvelle réforme des traités européens. Pour se limiter au cas de la France, le président Hollande ne serait pas très disposé à prendre le risque d'un nouveau traité qui devrait passer par l'étape de la ratification, et encore plus par celle du référendum. Si les contraintes du climat politique dans chacun des membres de la zone Euro permettent de comprendre la prudence des chefs d'Etat et de gouvernement, il n'en demeure pas moins que le statu quo du cadre institutionnel n'est pas une solution viable à long terme. La gouvernance de l'UEM montre en effet ses limites, tant du point de vue de l'efficacité des procédures que de la légitimité de ses contrôles.

La chancelière Merkel est peut-être le seul chef d'Etat et de gouvernement européen qui se soit déclaré à un moment favorable à une évolution des traités. C'était au Bundestag, le 18 décembre 2013. Néanmoins, Angela Merkel n'a jamais cherché à engager un débat sur ce sujet au sein de la société allemande. Son approche de la gouvernance européenne consiste plutôt à pointer du doigt des problèmes fonctionnels précis sans véritablement prendre le risque d'engager un débat politique sur l'avenir de l'UE. C'est une différence notable entre Angela Merkel et Helmut Kohl, son mentor en politique, qui traduit à la fois un changement générationnel d'attitude face au projet européen et une réticence plus grande à faire des institutions européennes un thème du débat politique national (je remercie ici Eric Bonse et Sabine von Oppeln d'avoir attiré mon attention sur cette question de l'approche strictement fonctionnelle d'Angela Merkel qui rejoint sans nul doute une attitude plus générale en politique).

Si l'on considère la question du lien futur entre UEM et Union Politique au sein de l'Europe, il conviendrait de régler plusieurs problèmes. Le premier est celui de la faiblesse de l'exécutif chargé de donner l'impulsion économique à la zone

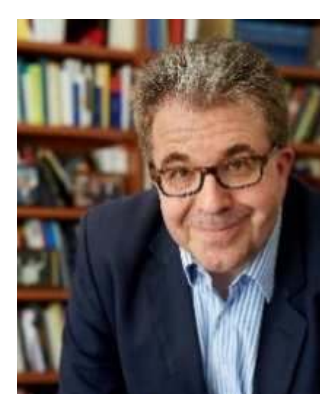

Christian Lequesne, directeur de recherche au CERI et professeur à Sciences Po Paris

Peu de gouvernements souhaitent une réforme des traités

Lien UEM/Union politique : plusieurs scénarios possibles 
Trois avantages en théorie : a/ intégrer dans les traités les acquis intergouvernementaux

b/ pouvoirs de contrôle accrus du Parlement européen

/ politisation de la gouvernance européenne
Euro. L'Eurogroupe n'est pas un organe doté de suffisamment de pouvoir de décision. II n'est pas présidé et donc représenté par une personnalité forte. Dans le domaine strictement monétaire, la Banque centrale européenne possède ce pouvoir de décision qui fait défaut à la gouvernance économique. En second lieu, les mécanismes de contrôle parlementaire de la zone Euro sont très faibles. II est anormal que les décisions de l'Eurogroupe échappent tant au contrôle à la fois du Parlement européen et des parlements nationaux. Pour l'avenir, trois scénarios ont été parfaitement identifiés par Thierry Chopin dans une Note de la Fondation Robert Schuman qui a été publiée en parallèle, en allemand, par la Deutsche Gesellschaft für Auswärtiges Politik (Chopin, 2014). Nous rejoignons tout à fait Thierry Chopin tant dans sa préférence pour une réforme de fond que dans son constat pragmatique que celle-ci aura du mal à être menée à court terme au profit de « réformettes » sans modification des traités. Quels sont les trois options en question?

\section{Scénario 1 : réforme des traités}

II s'agit dans ce cas de mettre en œuvre la procédure générale prévue par l'article 48 du Traité sur l'Union européenne. Elle consiste à réunir au plus vite une Conférence Intergouvernementale composée des 28 Etats qui doivent décider à l'unanimité de réformer le Traité sur l'Union européenne et le Traité sur le fonctionnement de l'Union européenne, puis de soumettre ces réformes à ratification selon les règles constitutionnelles respectives de chaque Etat.

En théorie, un tel scénario comporte plusieurs avantages. II permet de rationaliser tous les traités intergouvernementaux qui ont été négociés depuis 2008 en les intégrant dans les traités européens et donc de les soumettre pleinement au contrôle juridictionnel de la Cour de justice des Communautés européennes. On pense notamment au Traité sur le Mécanisme Européen de Stabilité entré en vigueur en 2012 et au Traité sur la stabilité, la coordination et la gouvernance entré en vigueur en 2013 dans 25 Etats membres - c'est à dire tous, sauf la Grande-Bretagne et la République tchèque (suite à un changement de gouvernement, la République tchèque s'apprête à signer et à ratifier le traité, mais en ne souscrivant pas à la « règle d'or ».). II s'agit du traité qui a introduit la fameuse obligation d'équilibre des finances publiques nationales plus communément nommée « règle d'or ». Une telle réforme aurait l'avantage de rapatrier dans le cœur des traités européens les mécanismes qui ont été négociés depuis la crise de manière ad hoc en dehors. On peut ainsi parler d'une démarche consistant à «communautariser » la gouvernance de l'UE en y intégrant les acquis qui se sont développés de manière strictement intergouvernementale. Cela ressemble à ce qui se produisit en 1997, lorsque le Traité d'Amsterdam intégra dans les traités européens l'acquis du Traité de Schengen sur la libre circulation des personnes né en 1985 en dehors des traités.

Le second avantage de cette réforme «complète » serait de pouvoir accroître les pouvoirs de contrôle du Parlement européen mais aussi des Parlements nationaux sur les supervisions économique et budgétaire. Actuellement, l'un des problèmes de l'UEM est précisément que la gouvernance économique échappe trop largement aux assemblées législatives et que cela sert d'argument fort aux partis populistes pour affirmer que l'UEM est une construction totalement dépourvue de légitimité.

Le scénario aurait enfin un troisième avantage : il créerait autour du thème de la gouvernance européenne une politisation obligeant les partis politiques de gouvernement à se positionner sur l'Europe dans les Etats membres. Force est en effet de remarquer, en 2015 , que les partis eurosceptiques - qui représentent $29 \%$ des sièges dans le Parlement européen issu des élections de mai 2014 ont face à eux peu de partis engagés de manière combative dans la défense du « plus d'Europe ». Les partis comme l'UMP et le Parti Socialiste en France, la CDU en Allemagne, le Labour en Grande-Bretagne ont une attitude d'évitement de la question européenne. Ils sont conscients (surtout lorsqu'ils gouvernent) 
que la contrainte européenne est inévitable dans la gestion de l'économie, mais en même temps, ils ne sont pas en mesure d'articuler un discours proactif à destination de leurs électeurs sur le déplacement du pouvoir de la scène nationale vers l'Europe. Ce vide de discours est très largement favorable à la montée des partis anti-européens. Pour prendre l'exemple de l'Allemagne, l'AfD (Alternative für Deutschland) - crédité de $7,5 \%$ des intentions de vote dans un sondage d'octobre 2014 (F.A.Z., 22-10-2014) profite aussi du manque d'entrain politique de la CDU/CSU et du SPD sur l'avenir de l'UE. Les partis anti-européens se nourrissent largement de l'approche "moins on en parle et mieux on se porte " des partis politiques traditionnels sur la gouvernance européenne.

Pour autant, le scénario de la réforme " complète " a peu de chance de voir le jour, car les gouvernements n'ont pas envie d'affronter les débats de ratification, qu'ils soient parlementaires et encore moins référendaires. II y a ainsi très peu de chances que la chancelière Merkel et le président Hollande prennent le risque d'un débat politique ouvert sur l'Europe avant l'année 2017 qui verra en France l'élection présidentielle et en RFA le renouvèlement du Bundestag.

\section{Scénario 2 : signature d'un nouveau traité intergouvernemental de gouvernance économique}

La deuxième option consiste à ouvrir une négociation, en dehors des traités communautaires, sur la signature d'un nouveau traité intergouvernemental de gouvernance économique. Elle aurait l'avantage de créer un débat politique en limitant a minima les risques de rejet par un référendum négatif d'un ou plusieurs Etat(s) membre(s). Un tel traité intégrerait dans un seul texte les instruments issus de la crise (Mécanisme Européen de Stabilité ; Traité sur la stabilité, la coordination et la gouvernance; Union bancaire). Avec l'intégration du MES, on pourrait ainsi rendre visible l'engagement des Etats à assurer une solidarité financière par des transferts vers les Etats de la zone Euro ayant des difficultés. Cela obligerait notamment à débattre en Allemagne de manière explicite de la question de la "Transferunion» ("union de transferts»). Un tel traité pourrait aussi créer des institutions propres à la zone Euro, en particulier un Président permanent de l'Eurozone et un Parlement de l'Eurozone composé à la fois de représentants du Parlement européen et des parlements nationaux.

Un tel traité serait signé simplement par les membres de la zone Euro et les pays aspirant à en devenir membres. Cela éviterait de gêner les pays qui, comme la Grande-Bretagne, ne souhaiteront par principe pas y participer. De même, il pourrait être envisagé, comme lors de la signature du Traité de 2012 sur la stabilité, la gouvernance et la coordination, que la ratification par une majorité d'Etats membres suffise à son entrée en vigueur.

On pourrait imaginer que les pays du Triangle de Weimar (Allemagne, France, Pologne) pourraient prendre l'initiative de ce traité, et affirmeraient ainsi le besoin de leadership de la construction européenne. Bien entendu, les partisans d'une UE " communautaire » n'aimeront pas l'idée d'un nouveau traité intergouvernemental, car il s'agit d'une solution institutionnelle qui néglige les traités européens (Traité sur l'Union européenne et Traité sur le fonctionnement de I'Union européenne). En même temps, le rôle renforcé qu'offre ce scénario aux parlements (européen et nationaux) ne permet pas non plus de parler d'une option purement intergouvernementale. Peut-être un tel traité illustre-t-il plutôt ce qu'Angela Merkel a appelé la "Unionsmethode " (méthode de l'Union) dans son discours au Collège d'Europe de Bruges du 2 novembre 2010. II s'agit d'un modèle de décision dans l'Union européenne qui est basé sur la coopération entre les gouvernements de l'Union européenne décidant par la voie du consensus. II n'est pas question dans cette méthode de proposition obligatoire de la Commission européenne, de vote à la majorité au Conseil des ministres et de pouvoir juridictionnel de la Cour de justice des Communautés européennes. Les critiques reprochent à cette "Unionsmethode » présentée par la chancelière Merkel d'affaiblir les institutions européennes et de ne pas contribuer à ré-
La méthode intergouvernementale limite les risques de rejet par référendum

Ratification à la majorité

Unionsmethode

prônée par A. Merkel 
Un gain d'efficience de la gouvernance...

... mais un pouvoir de contrôle réduit pour le Parlement européen duire la crise de légitimité démocratique de l'Europe en négligeant totalement le pouvoir de contrôle des parlements, à la fois européen et nationaux (voir Sarrazin/Kindler, 2012).

\section{Scénario 3 : améliorations à traités constants}

La troisième option, qui a le plus de chances de voir le jour, vise à régler la question de la gouvernance de l'UEM en favorisant des améliorations dans le cadre des traités existants et donc en évitant tout débat politique au profit d'aménagements fonctionnels. II est assez facile de démontrer, comme l'a fait la Commission dans ses propositions de novembre 2012, que plusieurs réformes de la gouvernance économique peuvent être opérées en n'introduisant aucune réforme formelle des traités européens. Avec cette option, il s'agit simplement de rendre plus efficaces les mécanismes de convergence budgétaire et fiscale existants (Semestre européen, Six-Pack, Two-Pack) en les assortissant d'un calendrier de mise en œuvre plus contraignant.

Il est également possible, sans réformer les traités, de créer un poste permanent de président de l'Eurozone chargé de représenter la zone Euro, en particulier dans les organisations financières internationales (Banque mondiale et FMI). En revanche, il y a peu de vraie possibilité avec ce scénario minimaliste de modifier en profondeur les pouvoirs de contrôle du Parlement européen et des parlements nationaux. Le choix du fonctionnalisme s'accompagne en effet logiquement d'une faible volonté d'accroître le contrôle démocratique de l'Europe, le principal étant de régler des problèmes techniques sans trop y associer les peuples.

Au FINAL, C'EST BIEN L'EVITEMENT DE LA POLITISATION qui constitue la principale limite du scénario de la réforme à traités constants. La réforme de la gouvernance économique de l'UE montre en effet que les gouvernements sont confrontés à un énorme paradoxe. D'un côté, ils refusent d'initier tout chantier ambitieux de réforme des traités qui conduirait forcément à devoir déléguer plus de pouvoir à un exécutif et à une institution parlementaire européens. De l'autre, ces mêmes gouvernements sont chaque jour défiés davantage par la montée des partis anti-européens qui, eux, n'évitent pas la politisation du thème européen, quitte à raconter souvent à leurs électeurs des contre-vérités (ou au moins des demi-vérités) sur la manière dont l'UE et la zone Euro fonctionnent.

Il faudra bien à un moment que les gouvernements assument une réalité : le meilleur moyen de combattre les eurosceptiques consiste à politiser de manière explicite le thème européen dans la sphère publique et non à le mettre sous le tapis. II est faux de penser que les citoyens sont incapables de comprendre et d'accepter les contraintes d'une coordination européenne renforcée des politiques macroéconomiques européennes. Ceux-ci sont prêts à entendre ces choses, à condition que les responsables gouvernementaux aient le courage de «monter au créneau » pour leur expliquer que dans le contexte d'interdépendance qui est celui de l'Europe - et plus largement du monde - il n'y a plus le choix de faire chacun sa « petite cuisine ».

\section{Indications bibliographiques}

- CHOPIN T., «Réformer l'Union européenne. Quelles méthodes ? Quels scénarios », Fondation Robert Schuman, Questions d'Europe, $n^{\circ} 320,7$ juillet 2014. Traduction en allemand: DGAPAnalyse, $\mathrm{n}^{\circ} 12$, juillet 2014

- Commission eURoPeEnNe, Blueprint for a Deep and Genuine EMU. Launching a European Debate, 28 novembre 2012

- DoutRIAUX Y., LeQUeSNe C., Les institutions de l'Union européenne, La Documentation française, Paris, 2013

- Guerot U., « Noces d'or franco-allemandes : le couple est-il fini ? », Politique étrangère, $n^{\circ} 4$, 2012

-SARRAZin M., KindLeR S.-C., «Brügge sehen und sterben - Gemeinschaftsmethode versus Unionsmethode ", Integration, Berlin, 3/2012

$\bullet$ www.bundesregierung.de. 